\title{
Entre Ducharme et le Mexique
}

Fragilité des transitions culturelles dans Drame privé de Michael Delisle

\section{Entre Ducharme y México. Fragilidad de las transiciones culturales en Drame privé [Drama privado], de Michael Delisle Between Ducharme and Mexico. Fragility of Cultural Transitions in Michael Delisle's Drame privé}

\section{Michel Nareau}

Volume 38, numéro 3 (114), printemps-été 2013

Michael Delisle

URI : https://id.erudit.org/iderudit/1018314ar

DOI : https://doi.org/10.7202/1018314ar

Aller au sommaire du numéro

Éditeur(s)

Université du Québec à Montréal

ISSN

0318-9201 (imprimé)

1705-933X (numérique)

Découvrir la revue

Citer cet article

Nareau, M. (2013). Entre Ducharme et le Mexique : fragilité des transitions culturelles dans Drame privé de Michael Delisle. Voix et Images, 38(3), 87-100. https://doi.org/10.7202/1018314ar

\section{Résumé de l'article}

Le roman Drame privé de Michael Delisle semble peu lié à l'ensemble de ses textes narratifs, tant priment dans ce récit un éclatement de la narration, un recours abondant aux marqueurs artistiques, un cadre urbain huppé. Ce texte fait d'emblée écho au roman L'hiver de force de Réjean Ducharme, par la mise en récit du milieu intellectuel montréalais, par la présence d'un narrateur s'exprimant au « nous » (pronom qui évoque la gémellité des protagonistes) et par une série de références à une jeunesse innocente. Or, une rupture survient dans le roman, qui fait bifurquer l'action vers le Mexique, transformant ainsi le cadre référentiel proposé. Dans ce changement se jouent le statut de la culture artistique mise en évidence et la capacité de cerner la valeur des pratiques culturelles observées. De la proximité, voire de la connivence, entre les artistes et les narrateurs à la lecture distanciée du tourisme de masse, se creuse un écart de sens, un vertige culturel qui fait l'intérêt du roman. Dans cet article, l'auteure s'attarde à la fonction de cette dérive des sens, aux conditions de lisibilité culturelle évoquées par ce travail de labilité, de même qu'à la signification de ce détour mexicain, qui s'appuie certes sur le tourisme dans la diégèse, mais qui est autrement littéraire, par l'appareil paratextuel qui recourt à des citations de nombreux auteurs latino-américains. Ce dernier point (intertextualité fécondée par la tension entre pastiche et déférence) permet enfin de cerner le rôle de Ducharme dans cette remise en cause des apports culturels.
Tous droits réservés (C Université du Québec à Montréal, 2013

Ce document est protégé par la loi sur le droit d'auteur. L’utilisation des services d’Érudit (y compris la reproduction) est assujettie à sa politique d'utilisation que vous pouvez consulter en ligne.

https://apropos.erudit.org/fr/usagers/politique-dutilisation/ 


\title{
ENTRE DUCHARME ET LE MEXIQUE
}

\author{
Fragilité des transitions culturelles \\ dans Drame privé de Michael Delisle* l
}

\author{
$++$ \\ MICHEL NAREAU \\ Collège militaire royal du Canada
}

Le livre est tenu par des mains

Couvertes d'Amérique ( $M A, 37)$.

En 1989, Claude Beausoleil fait paraître une anthologie de la poésie mexicaine aux Écrits des Forges ${ }^{2}$, lançant du coup une collection latino-américaine et un large réseau d'échanges littéraires entre le Mexique et le Québec. Cette anthologie signale certes l'intérêt nouveau du lectorat québécois pour les productions poétiques mexicaines, mais elle montre aussi qui au Québec possède une connaissance intime de l'espagnol et des œuvres dans cette langue, puisque de nombreux poètes québécois collaborent aux traductions. Michael Delisle est l'un de ceux-là . Déjà en 1987, il avait fait paraître un recueil de poésie, Les changeurs de signes, où l'espagnol perce le texte dans un jeu d'alternances codiques rapportées à la quête du sujet poétique. Ce dernier est en apprentissage de l'espagnol, et cette découverte de la langue de l'autre fait en sorte de déstabiliser son énonciation:

Y a-t-il un moment où devant le frelatement des matières l'on soit porté comme Lucie
Fontaine et moi à aller voir ailleurs dans des cultures étrangères ou même dans les
phrases de l'enfance dont ne subsiste souvent que le chant, si les choses autres ne
sont pas comme le français qui bute devant les limites du pouvoir-dire [...]. (CS, 11)

L'espagnol y est d'une part un plaisir langagier, une découverte de musicalité, une expérimentation du langage, transformant le réel en signes opaques, décalés ${ }^{4}:$ «Je

\footnotetext{
* Pour obtenir la liste des sigles utilisés dans cet article et les références complètes aux œuvres de Michael Delisle, voir p. 11. 1 Cet article s'inscrit dans le cadre d'un projet de recherche financé par le Conseil de recherches en sciences humaines du Canada (2012-2014), réalisé avec Maurice Demers de l'Université de Sherbrooke et intitulé «Mise en récit de l'Amérique latine au Québec: transferts de sens d'une autochtonie continentale, 1940-2010». 2 Claude Beausoleil (dir.), La poésie mexicaine. Anthologie, Trois-Rivières/Bordeaux, Écrits des Forges/Castor astral, 1989, 224 p. 3 La revue Estuaire, sous la direction encore une fois de Beausoleil, avait fait paraître en 1987 un dossier ( $n^{\circ}$ 46) sur la poésie mexicaine, dans lequel Delisle traduisait deux poètes (Samuel Ronzón et Frida Rodríguez Gándara). 4 On pourrait émettre l'hypothèse que cette logique du décalage par le traitement du langage est ce qui relie le mieux l'ensemble des pratiques génériques de Delisle. Ces phrases de Chose vocale
} 
dis : allá, está una mesa desocupada. Je scande les accents ${ }^{5}$.» (CS, 11) L'effet de dessaisissement y est recherché, jamais conçu comme perte ou anéantissement; au contraire, une jouissance des mots espagnols est perceptible dans le recueil. D'autre part, la langue espagnole fait surgir une liminarité culturelle, entre deux espaces rabattus l'un sur l'autre. Après avoir évoqué la station de métro Sherbrooke dans un poème en prose, tout en notant la rumeur sociale du lieu en espagnol, Delisle conçoit un autre court texte sur la même page, en écho et sur le mode mineur (graphiquement présenté comme s'il était une note de bas de page), qui projette le lecteur au Mexique:

\begin{abstract}
À l'heure de pointe, Mexico City refait surface. La foule me presse. Dans chaque wagon, une plaque hecho en la Pocatière. L'effet d'un toponyme à saveur locale. Pas strictement exotique. La Pocatière. Un effet d'accent. Je dis : effet. Le réflexe de passer outre. Il est presque cinq heures. Je trouve que c'est tard. (CS, 13; l'auteur souligne.)
\end{abstract}

Une telle expérience de l'interpénétration déstabilisante des codes et des référents est au cœur du roman Drame privé, que Delisle fait paraitre deux années plus tard, en juxtaposant la mémoire et le canon littéraire québécois à un effet d'exotisme mexicain autour de la question du statut du culturel dans les Amériques. Publié aux Herbes rouges, puis à Paris en 1990 chez P.O.L., le livre est reçu comme une "prose de recherche ${ }^{6}$ », une volonté de revenir «à l'essence de la phrase ${ }^{7}$ ", même s'il cède à l'occasion au "maniérisme, car il y a aussi une façon concise d'être maniéré ${ }^{8} »$. Est ainsi mis de l'avant le formalisme du roman, avec le jeu sur la langue, avec les ellipses dans une histoire rachitique par moments, avec ce que Delisle nomme «des personnages désincarnés ${ }^{9}$ ». Drame privé est lu, dès la réception, selon une logique de l'écart formel, ce qui est d'autant plus juste que les glissements entre récit et prose poétique s'installent dans l'œuvre à partir de l'expérience mexicaine. En regard des textes narratifs ultérieurs de Delisle, il semble en porte-à-faux, tant priment dans ce récit un éclatement de la narration, un recours abondant aux marqueurs artistiques, un cadre urbain chic et huppé, alors que la pauvreté du quotidien et du langage pour le montrer fonde ses romans néoréalistes.

Le roman de Delisle fait écho à L'hiver de force ${ }^{10}$ de Réjean Ducharme, par la mise en récit du milieu intellectuel montréalais, par la présence d'un narrateur

$$
+++
$$

auraient leur place dans ses romans de la banlieue, même si la critique distingue souvent la production romanesque de Delisle de sa production poétique: «M'accorder une ombre. Motel. L'autre avale une frite et suce ses doigts bruyamment. Simule pour amorcer. Je me regarde répondre.» $(C V, 5) \quad 5$ Voir aussi: «UNE AUTRE VILLE (mise en mémoire). Et bâtardement les langues se font, se défont, découverte sur découverte. Dans la modeste capitale du Guerrero, un imprimeur, un regard fatigué à la Borges, des paupières lourdes à la Jean Leduc; il veille près de sa rotative; son local n'est pas plus grand que ça et sa porte, canicule oblige, est ouverte. Nous passons devant, touristes aguichés par les exotismes, ahuris par les ressemblances. Nous ralentissons. Qu'imprime-t-il ?//En être à écrire ce que signifie partir pour New York, ville espagnole. Mañana por mañana. Ce que disent les villes, ce que pensent les langues qui circulent dans ces villes et donc que pensent ces villes.» (CS, 18) 6 Hugues Corriveau, «La phrase tragique», Trois. Revue d'écriture et d'érudition, vol. V, nº 3, 1990, p. 153. 7 Annie Lafleur, «Drame privé ", Aurélien Boivin (dir.), Dictionnaire des œuvres littéraires du Québec, t. VIII : 1986-1990, Montréal, Fides, 2012, p. 259. 8 Jean Basile, «Drame privé: un premier roman qui a du ton!», La Presse, 3 juin 1989, p. K6. 9 Michael Delisle cité dans Hervé Guay, «L'identité précaire», Le Devoir, 11 novembre 1995, p. D17. 10 Réjean Ducharme, L'hiver de force, Paris, Gallimard, coll. «Folio», 1984 [1973], 274 p. Élisabeth Haghebaert, analysant 
s'exprimant au «nous» pour avaliser une gémellité des protagonistes principaux. Or, une rupture survient dans le roman, qui fait bifurquer l'action vers le Mexique, transformant du coup le cadre référentiel proposé. Dans ce changement se jouent le statut de la culture artistique mise en évidence et la capacité de cerner la valeur des pratiques culturelles observées, dans un contexte qui devient continental et qui nous oblige à déterminer comment les références sont évaluées dans les Amériques, à partir d'un malaise exemplifié dès le détour ducharmien. De la proximité et de la connivence, toutes deux ambiguës, entre les artistes et les narrateurs à la lecture distanciée du tourisme de masse se creuse un écart de sens qui fait l'intérêt du roman. J'étudierai la fonction de cette dérive, les conditions de lisibilité culturelle évoquées par ce travail de labilité, de même que la signification de ce détour mexicain, qui s'appuie certes sur le tourisme dans la diégèse, mais qui est autrement littéraire, par des citations d'auteurs latino-américains. Ce dernier point (intertextualité fécondée par la tension entre pastiche et déférence) permettra de cerner enfin le rôle de Ducharme dans la mise en cause des apports culturels dans un cadre qui dépasse le Québec pour atteindre une réflexion continentale sur la liminarité culturelle.

\section{UN DÉTOUR DUCHARMIEN}

Réjean Ducharme n'est jamais nommé dans Drame privé. Aucun de ses livres n'est cité. Ducharme, à la surface du texte, brille par son absence. Pourtant, la structure du roman de Delisle répond à celle de L'hiver de force, dans un jeu qui rabat sur le célèbre écrivain fantomatique la démarche entreprise par les protagonistes de Delisle. La question devient alors : qu'apporte un tel rabattement? Avec des interrogations subsidiaires: quelle est l'ampleur de ce recyclage formel ? Que reprendil, que laisse-t-il de côté? On le sait, L'hiver de force, publié en 1973, est un grand roman sur le babillage social, sur la multiplicité des discours qui s'entrechoquent dans une société, mais qui surtout s'élaborent par le ressassement, l'accumulation. La vanité d'une originalité est toujours remise en cause par l'ironie et l'idéalisme du couple Ferron ${ }^{11}$ :

$$
+++
$$

l'influence de Ducharme sur le corpus québécois, écrit: «[Ducharme] a stimulé les parentés. En littérature, il y a ceux qui se réclament ouvertement de lui, qui lui font des clins d'œil, soit par le ton, les thèmes, les allusions, la langue, la désinvolture, l'impertinence ou le type d'univers qu'ils mettent en scène, par exemple Louis Hamelin, Gaétan Soucy, Sylvain Trudel, Marie-Sissi Labrèche, Jeffrey Moore, Guillaume Vigneault, Olivier Choinière, etc. » («Une narrativité logodynamique tendance destroy : les récents Ducharme», René Audet et Andrée Mercier [dir.], La narrativité contemporaine au Québec, t. I : La littérature et ses enjeux narratifs, Québec, Presses de l'Université Laval, 2004, p. 205) Delisle ne se trouve pas dans cette liste, et rien, en effet, ne semble associer ces deux auteurs. 11 Sur ces questions, voir Jean-François Chassay, «L'apache et le capitaliste. De la télévision à la poésie: le poète et ses textes dans les romans montréalais de Réjean Ducharme», L'ambiguité américaine. Le roman québécois face aux États-Unis, Montréal, XYZ éditeur, coll. «Théorie et littérature», 1995, p. 109-126; Élisabeth Nardout-Lafarge, Réjean Ducharme. Une poétique du débris, Montréal, Fides, coll. «Nouvelles études québécoises», 2001, 310 p. ; Élisabeth Haghebaert, "Techniques de brouillage idéologique dans L'hiver de force », Réjean Ducharme. Une marginalité paradoxale, Québec, Nota bene, coll. «Littératures», 2009, p. 155-171. 
Ce paradoxe [refuser de s'opposer au caractère normatif de la grammaire et de la taxinomie] n'est pas accessoire dans le récit, il se trouve au contraire au cœur de l'organisation du texte, car l'entreprise du récit (cette «vie enregistrée») procède aussi de la volonté de nommer les choses; le texte est donc une «Flore laurentienne» de la vie de Nicole et André, une description par laquelle ils espèrent toucher au plus près le sens de leur vie ${ }^{12}$.

Il y a certes de cette acuité abrasive de l'oreille assaillie par le tohu-bohu social chez Delisle, même si la première perception d'une relecture ducharmienne tient davantage dans la structure diariste double que dans la déstabilisation de la langue commune qu'opèrent les narrateurs.

Drame privé s'articule autour de la prise de parole d'un homme dans la vingtaine, proche du milieu artistique, mais qui gagne sa vie à microfilmer des documents. Il partage son existence avec Anne, diplômée en histoire de l'art, qui se contente d'un poste de secrétaire. Les deux fréquentent le milieu théâtral, par le biais d'Ali Blanchart, comédienne reconnue et amie d'enfance d'Anne, avec qui elle a fait équipe dans une bande de majorettes. Une telle proximité du milieu artistique (théâtre, performance, danse, peinture et photographie dans les dernières sections) - ce que le narrateur nomme sa connaissance du "socius des terrasses» $(D P, 40)$ - ne va pas d'emblée avec une commisération pour ces pratiques. Pourtant, le regard que jettent les deux jeunes branchés est assez cinglant, centré principalement sur une déconsidération pour les œuvres, les artisans et les spectateurs, soit en se moquant du décorum, des redites, des angoisses créatrices, soit en marquant leur propre détachement envers le sérieux investi dans ces démarches. Ainsi, en pleine représentation théâtrale, l'intensité dramatique est ravalée par une puérile discussion entre les deux spectateurs, pourtant présents pour encourager leur amie: "Anne me demande si ça va me tenter de manger chinois. Je fais signe que je ne sais pas. On nous fait "Tchh!" dans le dos. Elle me demande si on sort tout de suite. Je lui rappelle qu'on a promis d'aller voir Ali dans les loges. Nous attendons la fin en bâillant.» $(D P, 13)$ Pour contrecarrer cette inattention alors qu'ils ont à interagir avec les comédiens en coulisse à la fin du spectacle, les protagonistes rusent avec les codes du thêâtre: «- Dis-lui : “On sent vraiment que tu as mérité le droit de dire ce texte-là." Ça ne rate jamais. Oublie pas de faire une pause après "vraiment".» $(D P, 14)$ Cette intervention sarcastique aura des conséquences tragiques pour Luc, le comédien à qui s'adresse la phrase sibylline, puisque celui-ci sera assailli par le doute et fera des deux spectateurs inattentifs ses interlocuteurs éthiques privilégiés.

Cet exemple de désacralisation des arts ou de critique du sérieux avec lequel est investie l'arène culturelle le montre, les points communs abondent entre Drame privé et L'hiver de force. La présence parallèle de deux couples branchés, dont la prise de parole est déléguée à l'homme, laisse d'ailleurs entrevoir des situations d'intimité

$$
++
$$

12 Micheline Cambron, Une société, un récit. Discours culturel au Québec (1967-1976), Montréal, l'Hexagone, coll. «Essais littéraires », 1989, p. 168. 
où l'érotisme est évacué au profit d'une entente fusionnelle entre les partenaires. Dans chaque roman, le couple partage un regard dégagé, distant, moqueur à l'égard du milieu culturel montréalais, dont est répertorié le langage affecté, codé et stérile, captation ironique rendue possible parce que les deux duos sont composés d'ex-universitaires (beaux-arts ou histoire de l'art) confinés par choix dans des emplois subalternes de l'industrie culturelle (correcteurs d'épreuves, secrétaire, serveurs dans des bars branchés, documentariste, etc.). D'une telle posture périphérique, mais informée, la charge n'en est que plus mordante. Plus encore, les deux couples chérissent chacun une comédienne, Petit pois/la toune dans un cas, Ali Blanchart dans l'autre, même si elles incarnent le stéréotype de l'extravertie ampoulée et irresponsable.

Derrière la fréquentation du milieu culturel, les couples ont la volonté de se retirer du monde, d'habiter l'intimité d'une demeure en se coupant des rapports sociaux et des demandes communautaires qui viennent avec la nécessité d'être à l'affût des tendances. En ce sens, il n'est pas étonnant de voir surgir une scène de farniente au lit ${ }^{13}$ dans Drame privé, tant ce meuble incarne dans L'hiver de force le foyer à partir duquel la préhension du babil télévisuel peut être accomplie. Cette claustration dans la chambre à coucher, qu'elle soit liée à une coupure d'avec le système de consommation ou à un retranchement dans la sphère de l'intime, se conçoit autour d'un double pôle; d'une part, un plaisir actif émane du recours à cet habitacle, alors que le savoir livresque y acquiert un poids et un ordre à défaut d'un sens (Flore laurentienne et encyclopédie versus catalogue de peinture et manuels de neurologie $[D P, 94]$ ); d'autre part, ce refuge participe d'une ascèse matérielle délibérée («nous vivons ainsi dans l'angoisse d'une tentation » $[D P, 45])$ qui fait en sorte que l'argent est conçu uniquement dans un rapport à la dépense, à la combustion, avec la hantise de la thésaurisation.

De cette retraite ${ }^{14}$, à l'écart du tumulte urbain mais y participant bien malgré eux, les couples notent ce qui s'opine autour d'eux (publicités, chansons, marques de commerce) pour former une posture de «dissidents» $(D P, 109)$ à partir de ce bavardage et des observations colligées dans des cahiers d'écriture scolaires, Hilroy pour les Ferron, à «figure aztèque (tlaloc?) en frontispice» $(D P, 59)$ pour Anne et son conjoint. Dans ces objets industriels, déclassés ou appartenant au tourisme de masse, se retrouvent des expressions figées, des langues en contact et en confrontation, des titres d'œuvres artistiques, un magma agencé comme les entrées d'une quête d'authenticité ${ }^{15}$ toujours déjà compromise. Ne demeure possible qu'une position énonciatrice négative, exhumant, par annotations, la déréliction de ceux qui fuient les «drames privés» et refusent «d'effleurer le cœur des phrases. Ce serait jouer avec le feu» $(D P, 97)$.

$$
++
$$

13 «Elle [Anne] me parlait de literies douillettes dans lesquelles elle nous imaginait souvent, à l'abri du monde; elle parlait de "flanelles pêche" qui nous enturbanneraient les reins jusqu'à la grâce, de literie d'importation française; elle en parlait comme d'un paradis perdu avec dérision et douleur.» ( $D P, 45$; l'auteur souligne.) $\mathbf{1 4}$ «Les scotchs que je refuse aussi bien que les tailleurs d'Anne deviennent des affronts à l'art de vivre des créateurs. Nous sommes une tare sociale non pas parce que nous n'avons pas vécu, mais parce que nous refusons de vivre cette recherche que comprend la Dérive. » $(D P, 25) 15$ Le narrateur de Delisle «ne sai[t] pas encore comment» raconter «une histoire vraie» (DP, 97; l'auteur souligne). 
Si on ajoute une relation maniacodépressive au téléphone $(D P, 16)$ et à la consommation d'alcool, de drogue et de nourriture, il apparaît clairement que le modèle ducharmien est repris dans le roman de Delisle. Des années 1960 et 1970 contestataires, où la "Contre-Culture de Consommation, la CCC ${ }^{16}$ ", est à la fois exhaussée et désintégrée dans ses prétentions, ses mots d'ordre et ses élites bienpensantes (affirmation nationale, syndicalisme), on passe avec Delisle aux années 1980, sapées par l'individualisme, l'ambition économique, le carriérisme et l'essor d'une industrie culturelle tournée vers le marché international. Deux courts passages, où le narrateur de Drame privé évoque des performances artistiques, renvoient aux réactions d'André et de Nicole devant les taches jaunes de Laïnou ${ }^{17}$ : mépris, jalousie, critique féroce des conventions et des réputations artistiques. Le premier a lieu lors de la présentation d'Au secours ! de Paul Bédard : "Au premier geste de la performance de Paul Bédard, je me souviens, à mon grand désespoir, de l'avoir déjà vue, il y a deux ans. Il entre sur scène, s'assoit sur une chaise et ne bouge pas, ne dit rien, respire à peine pendant 45 minutes. Après ce temps, il se lève et sort.» $(D P, 20)$ L'extrait expose certes la redondance et l'incompréhension de la prestation, le jeu sur la performance et l'anticipation, ce que le narrateur nomme «la pragmatique de la représentation » $(D P, 19)$, mais il le fait de telle sorte que la critique du spectacle, que le terme «désespoir » entérine, est inscrite dans un débat culturel entre Luc et le narrateur, où la défense et l'illustration sont tenues par le narrateur qui, moqueur, «concède que c'était un peu long» $(D P, 20)$. L'ironie est alors autant dans la description du geste artistique que dans la réaction outrée du public, en faisant du savoir culturel l'enjeu du débat. Quand le diariste décrit ensuite la danse d'un ancien des beaux-arts condamné à faire des stripteases pour des bourgeoises anglophones de cinquante ans blasées, dans le plus pur style «chic colonial» $(D P, 44)$, c'est toute la portée du savoir culturel, des conditions concrètes de la création et de l'interprétation qui est conspuée dans une formule aussi distanciée que: "ça manque un peu de profondeur, je trouve» $(D P, 44)$.

Pourtant, les affinités des deux couples autour de leur posture de témoins culturels ambigus et dégagés - arbitrairement et temporairement - des modes sont limitées par des distinctions entre leurs parcours réciproques, différenciations à même d'inscrire l'écart entre l'effet de la parole communautaire de la Révolution tranquille et l'effet de désillusion des années postréférendaires. La langue employée diffère d'une œuvre à l'autre. Si L'hiver de force, en se constituant comme caisse de résonance désacralisante des aspirations de l'élite culturelle québécoise, doit passer par un hétérolinguisme qui puise à la langue populaire montréalaise, aux mots codés du milieu artistique, au joual de la rue, aux expressions anglaises des médias et à l'argot parisien des films ${ }^{18}$, la langue chez Delisle est davantage contrôlée, résistante à cette fusion des styles qui impose un dénominateur commun. L'ellipse y est la figure centrale, qui permet non seulement de tout ramener à la posture du narrateur, mais surtout de procéder à un retranchement des débats, des dialogues, pour les traiter avec

16 Réjean Ducharme, L'hiver de force, p. 189. 17 Ibid., p. 19-20. 18 Voir Aurélie Berthomieu, Plurilinguisme et satire dans L'hiver de force de Réjean Ducharme, mémoire de maîtrise, Montréal, Université du Québec à Montréal, 2011, 125 f. 
distance, par résumés équivoques et sarcastiques. La communication culturelle y est coupée court, renvoyée à une bulle distante. La diversité montréalaise émerge moins chez Delisle que chez Ducharme, même si l'espagnol, comme l'anglais, y trouve une place. C'est que l'atomisation sociale et l'intimité y sont mises en procès discursif, comme si la bulle culturelle s'était étanchéifiée par le succès international des années 1980. Les formes testimoniales employées dans Drame privé, tout comme les adresses, prennent plusieurs tournures, du récit réaliste à la lettre à quatre mains, en passant par le cahier intime et la prose poétique de voyage initiatique. Elles participent d'un besoin d'ancrage, de renouvellement de la communication culturelle à partir du moment où la liminarité du savoir artistique est reconnue et perçue comme un déclassement personnel. Une telle pratique peut se comprendre comme une quête du mot juste ${ }^{19}$, une recherche de légitimité de la parole, avec un protagoniste toujours au seuil de l'anonymat social - il n'est pas nommé - , de la dissolution de ses repères culturels.

Le détour ducharmien, en vertu de son caractère feutré, participe d'une logique de l'allusion qui circonscrit un malaise identitaire et culturel en reprenant un modèle québécois qui explore lui-même la problématique de la liminarité. En conservant la perspective ducharmienne sur les discours figés, mais en déplaçant l'objet de la critique du communautarisme vers l'ouverture néolibérale au monde, Delisle place la question de la mémoire et du canon problématiques au cœur de sa fiction, par le biais d'un des textes marquants du corpus québécois, en présentant une filiation inavouable, à tout le moins non avouée. Le séjour mexicain met en scène le témoignage du déficit culturel québécois, en le faisant bifurquer pour le sortir de l'ornière nationale et l'intégrer à un discours continental. André et Nicole, dans L'hiver de force, se remémorent à quelques reprises avec nostalgie leur séjour au Mexique, réalisé grâce au soutien financier du Conseil des arts du Canada. Ce voyage apparaît comme un sommet dans leur éducation culturelle, mais il n'a pas sa place dans la tentative d'épuisement du sens qui les occupe, parce que cette sortie du territoire communautaire ferait intervenir un autre axe du discours culturel. De leur côté, Anne et le narrateur, dans Drame privé, vivront ce dépaysement en temps réel, dans un journal de bord qui décrit la découverte de soi déstabilisante qu'est la confrontation avec l'altérité, dessaisissement qui met en jeu le savoir culturel dans des sociétés fondées sur la nouveauté et l'idée d'appartenir à un «faubourg de $1^{\prime}$ histoire ${ }^{20}$ », comme les autres habitants des Amériques.

$$
+++
$$

19 La lettre adressée à Luc, rédigée d'emblée par le narrateur mais annotée par Anne, signale que ce qui est à dire court le risque de passer outre, de manquer la cible, de négliger des détails importants et d'être en somme en deçà de l'expérience, de la quête décrite. D'ailleurs, ce désir d'exactitude est lié à la description de «l'été 1982 ", ce moment de dérèglement des sens où le couple s'est distancié de la société et de la culture en s'érigeant un rempart par l'alcool, les drogues, la consommation et l'enfermement. Le quotidien hors de la routine et la communion dans la marginalité ne peuvent se raconter dans des mots communs, ce qui oblige les deux personnages à un discours double et autocorrecteur, dont le narrateur conserve la trace par l'usage de l'italique. 20 Octavio Paz, Le labyrinthe de la solitude, traduit de l'espagnol par Jean-Clarence Lambert, Paris, Gallimard, coll. «nrf essais », 2002 [1950], p. 237. 


\section{LE MEXIQUE DE LA FÊTE DES MORTS}

Le séjour mexicain est évoqué à quelques reprises avant qu'il ne se produise et il est remémoré par la suite, ce qui a pour effet d'amplifier le caractère transformateur de ce dépaysement. Dès la première évocation, ce pays d'Amérique latine est appréhendé en fonction d'une double manière d'aborder le tourisme, l'une positive, l'autre négative: «Si je peux me libérer, nous irons, si je le veux, au Mexique, mais pas au bord de la mer, plutôt à l'intérieur des terres, dans les montagnes, si je veux.» $(D P, 30)$ L'extrait laisse entrevoir, par les précautions argumentatives réitérées, que le séjour ne va pas de soi, parce que la version touristique de masse axée sur la plage, le soleil, le «tout inclus» et l'exotisme bon teint est conspuée, l'hinterland mexicain ${ }^{21}$ apparaissant comme une zone authentique, inentamée, digne d'une entreprise de reconnaissance des signes spécifiquement mexicains, hors des flux touristiques conditionnés. C'est donc sous le signe de la rencontre de l'autre ${ }^{22}$ que s'élabore la mise en récit du voyage à Oaxaca, puisque les deux voyageurs sont à la recherche de «sensations paratouristiques» $(D P, 60)$. Il n'est donc pas étonnant, dans ce contexte, qu'ils s'établissent dans le quartier zapotèque de la ville, chez la sœur de la bonne de l'hôtel où ils ont atterri, pour mieux pénétrer ensuite dans la vie «authentique» de la ville. «Demain nous réglerons le cas des cartes postales» $(D P, 61)$, disent-ils, pour en finir avec cette posture d'étranger. La recherche d'authenticité les place ainsi en situation de manque à combler par le biais d'autrui, dépositaire d'un surplus de sens.

Le séjour mexicain est conté par fragments, à partir du moment où les deux protagonistes achètent chacun de leur côté un cahier. Le narrateur entame le sien, en livrant, sous forme d'un journal de bord, le récit de son arrivée à Oaxaca, capitale de l'État éponyme, phare moins touristique que Veracruz ou le Yucatán. En omettant le mois et l'année, le narrateur présente une version tronquée, sans repères temporels clairs de ce séjour, mais il insiste néanmoins sur la quotidienneté de sa pratique testimoniale, des entrées étant rédigées chaque jour, et à l'occasion plusieurs fois par jour, moins en fonction des événements qui se produisent (rares, de fait) qu'en vertu de l'ennui ressenti. Est ainsi mimé un séjour touristique, fait de découvertes, de reconnaissances d'archétypes culturels déjà intégrés, de rencontres, de conflits : «[L]e récit

$$
++
$$

21 Dans le discours sur l'américanité, cet espace reculé, difficile d'accès, parce qu'il évoquait la sauvagerie, les défis de la nature, a longtemps été envisagé comme une zone liminaire où se réalisaient les métamorphoses. L'hinterland est le lieu où s'effectue le scénario transformationnel de l'apprentissage de l'autre. Jean Morency, dans Le mythe américain dans les fictions d'Amérique. De Washington Irving à Jacques Poulin (Québec, Nuit blanche, coll. «Terre américaine», 1994, 258 p.), est celui qui a le mieux exploré cette figure. Les romans Utop de MarieChristine Arbour (Montréal, Triptyque, 2012, 209 p.) et Le postier Pasilla d'Alain Beaulieu (Arles/Montréal, Actes Sud/Leméac, 2010, 185 p.) sont deux illustrations récentes de ce scénario commun de la mise en récit de l'Amérique latine au Québec. 22 «Dans la continuité du xixe siècle, le récit des voyageurs reste en grande partie jusqu'à la fin de la Deuxième Guerre mondiale celui de la propagande et de la commémoration ethnocentrique, destiné à la réappropriation d'un patrimoine culturel et religieux. [...] Il en va tout autrement dans la seconde moitié du siècle, où l'on assiste, dans la littérature de voyage, à une importante remise en cause et en forme de l'autoreprésentation individuelle et collective, désormais marquée par une ouverture à une spatialité et à une temporalité différentes, mais surtout à des altérités multiples. » Pierre Rajotte, «Introduction », Pierre Rajotte (dir.), Le voyage et ses récits au Xxe siècle, Québec, Nota bene, 2005, p. 13-14. 
de voyage fictif ne doit pas son existence au déroulement d'un événement réel (le voyage). Il est davantage le fruit d'une vision du monde, vision qui passe toutefois par la nécessité de faire du déplacement le centre d'un processus visant à recomposer le réel ${ }^{23}$. » La ponctuation linéaire de la narration participe d'un travail de l'ellipse et de la mise à distance qui était auparavant présent dans le récit du tumulte montréalais, renvoyant de ce fait le Mexique au Québec.

Dans Le récit de voyage canadien-français au dix-neuvième siècle. Analyse du succès d'une pratique littéraire, François Couture signale qu'une des caractéristiques du récit de voyage est de procéder à une pratique de la substitution par comparaison : «Le narrateur rattache les lieux visités à un point de repère (généralement sa patrie), il effectue une réflexion comparative plus ou moins étendue ${ }^{24}$.» Dans Drame privé, c'est par le biais de la jeune zapotèque Prisca que cette liaison s'établit. À travers elle, c'est l'exotisme jugé authentique qui semble atteint; surgit alors l'innocence de l'enfance - autre trait ducharmien? - , le naturel amérindien, la communion verbale, la connivence des gestes, la fraternité de la rencontre gratuite. Elle devient le vecteur d'un retour sur soi, d'une plongée dans le passé : «Il y eut ensuite un monologue des plus curieux où Anne a expliqué à Prisca - en espagnol! - la différence entre les Cavalières de la Rive-Sud et les Châtelaines de Laval. Prisca a bien aimé las Caballeras de la Ribera del Sur.» $(D P, 62)$ Elle assure le passage entre deux mondes, deux temporalités, elle est un truchement qui rapproche ${ }^{25}$ et donne accès à l'univers amérindien sous le signe de l'imaginaire: "Ce sont souvent les petits détails qui retiennent mon imagination. Les pyramides de Monte Alban sont impressionnantes et le site est célèbre, mais ce qui me rend songeur c'est, bien plus, par exemple, Prisca, notre petite voisine zapotèque qui vend sa ménagerie d'onyx aux touristes du zócalo (la place centrale).» $(D P, 61)$ Cet extrait fait de l'amérindianité le moyen de s'éloigner du tourisme de masse - où les voyageurs se contentent de l'achat d'objets exotiques sans accéder au savoir local - par le biais d'une amitié précaire avec une enfant. Le rôle attribué à Prisca montre l'ambiguité du savoir culturel sur le Mexique, puisque cette connaissance ne passe que par le truchement ethnographique d'une authenticité forgée à même l'idée d'une origine, d'une naturalisation.

Anne et le narrateur se détournent des sites ${ }^{26}$ au profit de la rencontre d'abord parce qu'ils maîtrisent l'espagnol ${ }^{27}$, qu'ils peuvent établir un contact, mais ensuite

$$
++
$$

23 Jean-Pierre Thomas et Marie-Élaine Bourgeois, «Du voyage dans la fiction au récit de voyage fictif», Pierre Rajotte (dir.), Le voyage et ses récits au XXe siècle, p. 282. Ils ajoutent: «Le récit de voyage fictif polarise l'accent sur le déplacement lui-même et sur le sens transformationnel véhiculé par celui-ci.» Ibid., p. 308. 24 François Couture, Le récit de voyage canadien-français au dix-neuvième siècle. Analyse du succès d'une pratique littéraire, mémoire de maîtrise, Sherbrooke, Université de Sherbrooke, 1997, f. 21.25 "L'Amérique est un village. Tout finit par se savoir.» $(D P, 31)$ L'expression revient $(D P, 112)$, après que le narrateur eut évoqué le Mexique. 26 «Pour plusieurs par exemple, l'approche la plus naturelle est également la plus culturelle: c'est visiter les ruines [...] non pas dans leur existence concrète présente, mais comme reliquat d'un passé prestigieux [...]. Ce qui est intéressant, ce n'est ni l'agencement des pierres, ni leur usure, c'est ce dont elles sont le signe et le vestige, leur message historique. » Pierre Rajotte, «Aux frontières du littéraire. Récits de voyageurs canadiens-français au XIXe siècle», Voix et Images, vol. XIX, no 3, printemps 1994, p. 554-555. 27 La langue espagnole est abondante dans cette section du roman: «taquerias» $(D P, 61)$, «cantor» $(D P, 69)$, « recette mixtèque » $(D P, 69)$, « anciano » $(D P, 72)$, «mañana » $(D P, 73)$, « Juárez», «Cortez» $(D P, 75)$, etc. 
parce qu'ils sont hors circuit, qu'ils ont pris la voie dégagée des poncifs du voyage. Dans Drame privé, contre la logique des ruines, des traces, d'une anhistoricité de la culture amérindienne renvoyée à une origine qui est toujours liée à une noncontemporanéité ${ }^{28}$, les deux voyageurs refusent le récit livresque (du guide de voyage) et se laissent au contraire orienter par celle qui est là à jouer avec sa pièce de monnaie, sans qualification autre que celle d'incarner la promesse d'un présent. Cet être-là mexicain est accessible par décalage culturel et transforme le voyage:

\begin{abstract}
Demain, [Anne] retournera dans la région de Monte Alban pour photographier la végétation, pas nécessairement des ruines, juste «de la feuille verte». Je n'aime pas marcher dans la jungle. Elle ira sans moi. Je revisiterai peut-être le musée. On fait vite le tour de Oaxaca. Mais c'est bien de revoir les choses plusieurs fois, ça raffine les souvenirs. $(D P, 71)$
\end{abstract}

Tourner le dos aux ruines, à ce que Pierre Rajotte nomme «le processus de la transfiguration culturelle ${ }^{29} »$ de l'espace autre, c'est précisément creuser un écart par rapport aux modèles acquis. Or, rien n'est en mesure de combler le manque ressenti à ne plus se fier aux préconçus du tourisme de masse, et il n'est, dès lors, pas étonnant que cette bifurcation du séjour ait de nombreuses conséquences. Les ruines sont des zones balisées de confrontation aux morts; toutefois, hors de ce schéma, les spectres seront partout autour du couple, provoquant une rupture, une transformation de soi et une incapacité à inscrire sa propre histoire dans son journal. À partir de la visite d'Anne dans la jungle, la cohésion du couple se dégrade, jusqu'à la rupture au retour du Mexique. Un tel effilochage trouve à s'articuler dans le journal par le passage du récit au poème, comme si la langue se retournait sur elle-même pour porter la perte de repères, pour tracer les marques d'un délitement.

Le pays de la fête des Morts est dès lors associé à un dépérissement, dans la mesure où l'expérience de l'altérité y est perçue comme un moment où le couple perd pied. La traversée de la jungle qu'entreprend Anne, et qui ne nous est pas rapportée par le narrateur, lequel se met à ce moment à multiplier les ellipses, peut être envisagée comme une métamorphose. Anne part avec l'idée de prendre des photos, de cadrer le vert, de tirer de la magnificence du paysage et probablement de l'exubérance du passé autochtone un témoignage clair, pelliculé. Dans la forêt, elle se perd, elle trébuche sur son passé, elle s'enivre et perd contact avec le réel et son compagnon. Elle est retrouvée plus tard, délai rendu par l'attente fiévreuse du narrateur, déboussolé devant ce qu'il perçoit comme une désertion. Ainsi, pour les deux, l'expérience de la jungle est un moment de perte de soi, de mort symbolique, ce qu'ils sentent, mais différemment :

28 Sylvie Vincent et Bernard Arcand, L'image de l'Amérindien dans les manuels scolaires du Québec ou Comment les Québécois ne sont pas des sauvages, Montréal, Hurtubise HMH, coll. «Cahiers du Québec. Cultures amérindiennes ", 1978, 334 p. 29 Pierre Rajotte, avec la collaboration d'Anne-Marie Carle et de François Couture, Le récit de voyage au XIX $X^{e}$ siècle. Aux frontières du littéraire, Montréal, Triptyque, 1997, p. 96. 
À bord du vol de retour d'Oaxaca, Anne avait fini par accepter de jeter un coup d'œil sur mon journal où était inscrite une approximation un peu mystique de ce qui s'était révélé à moi sous cette foudre d'émotion. C'était souvent du poème mais je n'avais rien d'autre qui s'approchait autant du réel. Et j'espérais qu'elle pourrait y trouver un élément ou deux pour saisir mon état. Elle m'avait rebalancé les cahiers bêtement, avec comme unique commentaire : "J'aime pas ça, on dirait que je meurs à la fin. » C'est à cet instant que j'ai décidé d'abandonner. Je ne voulais plus convaincre personne de quoi que ce soit. $(D P, 106)$

Incompréhension, désarroi, abandon, conscience d'un changement subi, les motifs de la rupture, de la mise à mort de soi se font pressants. Le séjour mexicain, que les deux jeunes branchés s'offrent pour mettre à distance leur insignifiance culturelle et leur travail aliénant de subalternes, ne remplit ni la promesse du dépaysement, en les renvoyant à leur chute de 1982 dans le repli sur soi, ni celle de la régénération, puisque la mort de soi éprouvée au contact de l'autre ne se répercute pas en une renaissance. Le chemin du retour est un calvaire, dont la route mènera le narrateur jusqu'en Italie, dans une volonté de s'appuyer sur des vestiges culturels forts, plus forts que ceux rencontrés dans le milieu artistique et littéraire montréalais et dans la jungle mexicaine.

\section{PLONGÉE DANS LE MALAISE CULTUREL}

Drame privé, à la suite du voyage mexicain, perd de sa linéarité et de son unité temporelle. Un hiatus important advient dans la narration, période dont le lecteur sera tenu à une certaine distance. Le couple formé du narrateur et d'Anne a éclaté, le groupe des aficionados culturels dans lequel se trouvaient Luc et Ali ne se voit plus. Le narrateur s'est «tapi» $(D P, 94)$ dans un appartement quelconque «de la banlieue nord » $(D P, 94)$, dans une routine réconfortante et avilissante ${ }^{30}$, parce qu'il est submergé par ce que son "entourage nommait à voix basse "le Mexique" ( $D P$, 93-94).

Profitant d'un rare moment de vacances, il se rend à Venise, où il tombe par hasard sur Luc, qui travaille désormais dans le domaine de la publicité, voie de sortie des belles gueules du milieu thêâtral. Malgré le vernis d'un faux naturel que porte Luc, le narrateur demeure sur ses gardes, dubitatif vis-à-vis de ce lointain passé et de la sérénité feinte de son ancien ami: «Je lui dis, pour le tester, qu'il y a au Mexique des régions où les fantômes circulent librement, avec tout le naturel du monde, des régions où "les choses qui ne se disent pas" sont des choses entendues depuis toujours.» $(D P, 96)$ Le Mexique, espace initiatique et d'authenticité, est porté par un mystère, une métamorphose envisageable - «ces quelques instants où j'avais cru entrevoir le versant caché du monde» $(D P, 94)$ - qui renvoie ce lieu à un second

$$
++
$$

30 Ce rapport complexe à la routine caractérise l'œuvre de Delisle, notamment la nouvelle «Eau de Javel», publiée dans Helen avec un secret et autres nouvelles. 
«été 82 », période de mort à soi, de renaissance, où la mémoire opaque des choses remonte en révélant des secrets au cœur des phrases, rendant la langue suspecte et traversant les êtres, les choses, les relations pour faire éprouver le sens du dérisoire, du dépouillement de soi. Luc, à qui s'adresse le témoignage, "ne passe pas le test» $(D P, 96)$. Le Mexique, dans son étrangeté, est un rite de passage partagé entre initiés, dont Anne et le narrateur sont les légataires autodésignés. Contre les abîmes de ce savoir privé demeure le consensus culturel de la mode, les effigies publicisées, les grandes marques, qui ouvrent la surface fade du quelconque : «Ā cet instant, j'ai eu le génie d'enchaîner sur les vestons Armani.» $(D P, 96)$

Le recours aux vêtements comme armure indique une habitation-refuge à se constituer: «[N]ous étions venus à croire qu'il y avait un lien entre la rigidité des tenues et la protection nécessaire pour affronter le quotidien.» $(D P, 48)$ Le retranchement participe d'une réaction puérile du fait de son objet (les vêtements, la mode, la superficialité), mais la nécessité de contenance qui y est associée fait de la tenue vestimentaire le point nodal d'une appréhension de la culture par la mode, où tout risque de tomber dans l'obsolescence, le déclassement. La parure permet le classement, le positionnement de soi, mais au prix de sa propre destitution à terme. La rigidité comme exigence culturelle est alors une posture, une manière de se cadrer dans le monde, dont les canons sont déterminés dans un sous-groupe de deux, ne répondant qu'à une contingence, sans principes partagés. Ce jeu pour se bâtir une ossature culturelle mène à un univers étanche de privilégiés.

Dans la scène de la fête finale, Anne et le narrateur renouent, après une longue séparation. Ils se retrouvent, mais un malaise les enveloppe, trouble amplifié par le brouhaha de la fête et de ses conventions, puis par l'arrivée d'Ali et de Luc, les témoins d'une époque d'équilibre perdu et férocement recherché. Ils ne savent pas comment rétablir le dialogue innocent des années passées, et chacun se construit un interrogatoire à plaquer sur l'autre, questionnaire qui débute par la même clé de voûte (« D'après toi, est-ce que le monde a un sens ?» $[D P, 108,111]$ ), geste qui témoigne à la fois d'une affinité et d'un refus de communauté autre que celle d'une gémellité autosuffisante. Ce geste n'est que l'écho décalé de ces cahiers achetés au Mexique simultanément $(D P, 59)$ qui catégorisaient pour eux le bon goût de ceux qui se situent au-dessus «de l'hippodrome à touristes ${ }^{31}$ ", capables de séparer le bon grain de l'ivraie, même dans une culture millénaire qui demeure relativement opaque aux protagonistes.

Si l'expérience mexicaine tourne au cauchemar, si elle se limite, dans un de ses rares moments positifs, à la rencontre d'une fillette vendeuse de gommes à mâcher, c'est qu'elle marque une des limites du savoir sur l'autre. Le narrateur demeure en marge en ne pénétrant pas dans la jungle, en ne procédant pas au scénario transformationnel. Les échos futurs à propos du Mexique ont beau insister sur les fantômes et la métamorphose, il se sait à l'écart de cette culture, comme s'il n'avait pu que tâter

$$
++
$$

31 L'expression de Bruno Hébert (Le jeu de l'épave, Montréal, Leméac, 2005, p. 89) s'applique à une dérive identitaire dans un Mexique porté par l'héritage maya. 
une altérité qui l'attire, mais dont il est inapte à saisir l'importance. Une amertume couve sous ses évocations mexicaines, une sensation d'avoir échoué à saisir le «cœur de la phrase» $(D P, 9)$ mexicaine, malgré l'introspection et la connaissance de l'espagnol.

Pour pallier cet échec de sens, le narrateur tente une dernière fois de renouer avec ses anciens amis, avec Anne, avec la culture montréalaise, mais en procédant à des rabattements de sens et de référents qui joignent le tohu-bohu métropolitain à la culture mexicaine, et plus largement latino-américaine, sous forme de citations insérées dans le texte ayant la même vertu d'habitacle que les vêtements d'Anne et de Luc. Si les allusions culturelles abondent dans le roman, en raison de la position d'observateur défendue par le couple durant la première section du récit, qui s'appuie sur le modèle caustique de L'hiver de force, elles prennent une nouvelle tournure dans le septième chapitre («La somme des fatalités» $[D P, 99])$, placé sous l'autorité de Jorge Luis Borges, dont la citation («nous verrons avec notre corps tout entier» $[D P, 101]$ ) insiste sur le caractère senti des savoirs. Ce chapitre est sous-divisé en sections distendues, qui reviennent en boucle sur le désarroi du narrateur, son malaise à renouer avec une culture qu'il tient à distance, puisqu'il a conscience d'un manque d’héritage, de lien au passé:

Je sirote un expresso dense et brûlant. Les garçons ici travaillent avec un «art» et une «façon de faire» qui semblent leur avoir été transmis par leurs ancêtres. Personnellement, je n'ai jamais eu ce sentiment. Je dois bien avoir des ancêtres. Je remonte mentalement ma filiation. $C^{\prime}$ est si loin derrière, tout ça. $(D P, 94)$

Il aimerait porter avec naturel les traces d'un savoir ancestral, d'un «art de faire » légué, qu'il reconnaît à Luc et aux Vénitiens. Chacune des sections s'amorce par un exergue, béquille littéraire de celui qui ressent sa marginalité socioculturelle et qui se sait néanmoins porteur d'une connaissance livresque qui ne trouve aucune place dans son quotidien de manutentionnaire. Se succèdent alors Claude Beausoleil, Octavio Paz, Dante et James Sacré, dans un éclectisme certain. Si le passage citationnel se fait de l'Argentine à la France, en passant par le Québec, le Mexique et l'Italie, il mime en bonne partie le parcours du narrateur, tout en accordant une large place à un corpus latino-américain - auquel il faudrait greffer Beausoleil en raison de son travail de passeur culturel, qu'il effectue de manière contemporaine au roman - qui n'est pas encore bien connu au Québec et beaucoup moins naturalisé que le français et l'italien.

La citation de $\mathrm{Paz}^{32}$ indique clairement un travail sur l'exiguité de la culture, sur le sentiment d'exister à la lisière du monde, dans une périphérie culturelle. Il s'agit d'un constat effaré de sa propre petitesse, de l'immensité du récit déjà écrit dans lequel sa place n'est qu'aléatoire, dépendante d'une volonté extérieure à la sienne. Le narrateur, celui dont l'identité n'est ni formée ni reconnue, participe de cette insécurité ; il la traîne dans ses jugements sur les autres, soit sur le mode d'une certaine

$++$

32 «Je pensai que l'univers était une conversation entre des êtres immenses... Quel pouvait être le mot dont je n'étais qu'une syllabe? Qui le disait à qui ?» $(D P, 110)$ 
jalousie, soit sur celui d'une incapacité à coïncider avec les traces de grandeur (monde maya, milieu thêâtral, canaux vénitiens) qu'il croise et qui l'affectent en lui révélant son «labyrinthe de solitude», son incomplétude, sa fragilité.

Drame privé est alors le roman d'une quête de savoir, d'une plongée dans la langue, «au cœur de la phrase», pour retrouver des moments de connivence qui assurent un refuge contre une culture qui échappe aux protagonistes. Le séjour mexicain, parce qu'il offrait un autre départ possible, une stratégie différente de celle de l'ironie ducharmienne, a été vu comme le théâtre d'une métamorphose, mais qui ne remplit pas les promesses du Nouveau Monde. Pourtant, il y a, dans les suites de cette rencontre ratée avec l'altérité zapotèque, la reconnaissance d'un malaise culturel continental, ce que conforte le recours aux intertextes de Borges et de Paz. Dans son célèbre essai Le romancier fictif ${ }^{33}$, André Belleau notait le conflit des codes qui travaillait le corpus québécois, tiraillé entre une expérience américaine et des canons européens. Drame privé, en passant de Ducharme au Mexique, élargit ce conflit et montre que la fragilité culturelle dont le roman québécois porte le témoignage est un passage obligé de la prise de parole américaine au sens continental, parce que cette dernière procède d'une filiation problématique, d'un temps sans réelle continuité affichée, de cadres référentiels disparates et d'un espace qui se dérobe constamment ${ }^{34}$. Le détour ducharmien contribue à défaire la valeur culturelle de l'expérience de la scène montréalaise des protagonistes, alors que le Mexique révèle l'ampleur de leur excentrement et des contraintes qui les marginalisent malgré leur démarche vers l'authenticité. L'expérience de vacillement des signes que présente le récit de la chute du narrateur et d'Anne révèle la profonde concordance de l'exiguïté des assises culturelles des Amériques, ce que Fernando Ainsa, Leopoldo Zea, Édouard Glissant, Carlos Fuentes et Octavio Paz ${ }^{35}$ avaient vu pour l'Amérique latine et que Delisle reprend à son compte, pour inscrire un drame existentiel montréalais dans une logique de marginalité qui concerne toutes les collectivités américaines.

33 André Belleau, Le romancier fictif. Essai sur la représentation de l'écrivain dans le roman québécois, Québec, Nota bene, coll. «Visées critiques», 1999 [1980], 229 p. 34 J'ai développé longuement ces trois nœuds identitaires des Amériques (espace, temps et références problématiques) dans mon essai Double jeu. Baseball et littératures américaines, Montréal, Le Quartanier, coll. «Erres Essais», 2012, 395 p. 35 J'ai déjà évoqué la réflexion d'Octavio Paz sur l'excentrement culturel latino-américain. Il est rejoint là-dessus par l'auteur martiniquais Édouard Glissant, pour lequel les Antillais "n'ont jamais fréquenté leur temps historique, [ils l'ont] seulement éprouvé» (Le discours antillais, Paris, Gallimard, coll. «Folio. Essais», 1997, p. 344). Voir aussi Leopoldo Zea, L'Amérique latine face à l'histoire, traduit de l'espagnol par Jean A. Mazoyer et Jean Martin, Paris, Lierre \& Coudrier, 1991, 356 p. ; Carlos Fuentes, Le sourire d'Érasme. Épopée, utopie et mythe dans le roman hispano-américain, traduit de l'espagnol par Ève-Marie et Claude Fell, Paris, Gallimard, coll. "Le messager», 1992, 345 p. 